\title{
High prevalence of Pfmdr-1 N86 and D1246 genotypes detected among febrile malaria outpatients attending Lira Regional Referral Hospital, Northern Uganda
}

\author{
Emmanuel Achol ${ }^{1,3}$, Stephen Ochaya ${ }^{2}$, Geoffrey M. Malinga ${ }^{1,4}$, Hillary Edema ${ }^{3}$ and Richard Echodu ${ }^{1,3^{*}}$ (D)
}

\begin{abstract}
Objective: To determine the prevalence of Plasmodium falciparum multi-drug resistant gene-1 (Pfmdr-1) N86Y and D1246Y genotypes among febrile malaria outpatients attending Lira Regional Referral Hospital, Uganda.

Results: Overall, $92.3 \%(n=48 / 52)$ and $90 \%(n=45 / 50)$ of the parasites detected carried the wild type alleles $1246 \mathrm{D}$ and N86, respectively. Only $7.7 \%(n=4 / 52)$ and $10 \%(n=5 / 50)$ of these P. falciparum isolates carried the Pfmdr-1 mutant alleles $1246 \mathrm{Y}$ and $86 \mathrm{Y}$, respectively. Our results show high prevalence of wild type alleles N86 and D1246 in $P$. falciparum isolates from Lira Regional Referral Hospital, which could translate to a decreased sensitivity to artemetherlumefantrine. Continued monitoring of prevalence of single nucleotide polymorphisms is warranted to timely inform malaria treatment policies and guidelines.
\end{abstract}

Keywords: Malaria, Pfmdr-1, Mutation, Drug resistance, Genotypes, Prevalence, SNPs, Resistance

\section{Introduction}

Malaria mainly caused by an intracellular protozoan Plasmodium falciparum remains a serious infectious disease with high morbidity and mortality worldwide. In 2018, the sub-Saharan Africa accounted for $92 \%$ and $93 \%$ of the global malaria cases and deaths, respectively, with $4 \%$ of the total world burden occurring in Uganda [1]. Uganda has a stable and perennial malaria transmission in $90-95 \%$ of the country, with a low and unstable transmission especially in the highlands usually with potential for epidemics [2].

In the absence of effective malaria vaccines, early and successful treatment is vital in reducing morbidity and mortality. However, the emergence and spread of antimalarial drug resistance has contributed to a global increase in malaria related deaths [3]. Previously, resistance to

*Correspondence: richardechodu2009@gmail.com

1 Department of Biology, Faculty of Science, Gulu University, P.O. Box 166 Gulu, Uganda

Full list of author information is available at the end of the article chloroquine (CQ) and sulfadoxine-pyrimethamine (SP) hampered malaria control efforts resulting in the adoption of artemisinin-based combination therapy (ACT) as the standard treatment for uncomplicated $P$. falciparum malaria $[4,5]$. ACT consists of a rapid-acting artemisinin derivative with a longer-acting partner drug that clears parasites not eliminated by the artemisinin component and limits selection of artemisinin resistance [6, 7]. The re-infecting parasites are exposed to slowly declining drug concentrations during clearance of the long-acting partner drug and a shorter post-treatment prophylactic effect could reduce the ACT to an artemisinin derivative monotherapy [8]. In 2004, artemether-lumefantrine (AL) was adopted as the first line treatment for uncomplicated malaria in Uganda, with artesunate/amodiaquine (AS/ $\mathrm{AQ}$ ) as its alternative [9].

Mutations in $P$. falciparum genes, identified as single nucleotide polymorphisms (SNPs) are markers of antimalaria drug resistance which allow for the monitoring of declining drug susceptibility in parasite populations [1012]. For partner drugs in the national treatment regimens 
$\mathrm{AL}$ and $\mathrm{AS} / \mathrm{AQ}$, increased risk of treatment failure and decreased susceptibility to lumefantrine in vitro are associated with N86, 184F, D1246, K76 alleles of Pfmdr$1[11,13,14]$. AQ resistance is associated with $P f m d r-1$ alleles 86Y, Y184 and 1246Y; P. falciparum chloroquine resistance gene $(P f c r t)$ alleles $76 \mathrm{~T}$ and SVMNT while CQ resistance is associated with $86 \mathrm{Y}$ and $76 \mathrm{~T}$ alleles of Pfmdr-1 and Pfcrt genes respectively [13, 15]. Over the years, changes in malaria treatment practices have resulted in changes in the above markers of resistance. This study aimed at assessing the prevalence of $P f m d r-1$ $\mathrm{N} 86 \mathrm{Y}$ and D1246Y polymorphisms in P. falciparum isolates collected from malaria outpatients at Lira Regional Referral Hospital (LRRH), Uganda. We observed a high prevalence of wild type alleles N86 and D1246 and low prevalence of the mutant type alleles $86 \mathrm{Y}$ and $1246 \mathrm{Y}$, which could translate to a decreased AL sensitivity in the region.

\section{Main text \\ Methods}

\section{Study sites and population}

This study was conducted in Lira regional referral hospital (LRRH), Lira district, Northern Uganda $\left(1^{\circ} 21^{\prime} \mathrm{N}\right.$ $02^{\circ} 42^{\prime \prime} \mathrm{N}$ and $32^{\circ} 51^{\prime \prime} \mathrm{E} 34^{\circ} 15^{\prime \prime} \mathrm{E}$ ), a high-endemic malaria area, located approximately $340 \mathrm{~km}$ north of Kampala, covering an area of approximately $1326 \mathrm{~km}^{2}$. Malaria is present throughout the year, with seasonal peaks of transmission coinciding with the rains (March-May and September-December). LRRH is the only regional referral facility in the sub-region, offering both specialized clinical cares, including HIV/AIDS and general clinical services. The study population consisted of febrile malaria clients attending the antiretroviral therapy (ART, 251 patients) and out patients' department (OPD, 520) clinics. Patients diagnosed with $P$. falciparum malaria between April and July 2015 based on Giemsastained thick and thin blood films were included in the study after obtaining informed consent. Inclusion criteria were individuals who presented with fevers and had taken anti-malarial drug ACT at least once during the 12 months prior to the study.

\section{Sample collection}

Fingerpick samples of blood spots were collected on chromatography filter paper (Lasec, Munktell TFN), airdried, labeled and stored at $-80^{\circ} \mathrm{C}$ in plastic envelopes containing silica gel until further processing. Plasmodium falciparum microscopically positive samples were used for this study and all patients diagnosed to have malaria infections were treated with artemether-lumefantrine (Coartem).

\section{DNA extraction, PCR and RFLP assays}

Plasmodium falciparum DNA was extracted from all filter paper dried blood samples using TRIzol reagent LS (Epigenetics USA) as per manufacturer's protocol. Outer and nested PCR were performed for codons 86 and 1246 of $P f m d r-1$ on all microscopically positive samples following the protocol developed by Humphreys et al. [16], followed by restriction length polymorphism (RFLP) digestion using restriction enzymes as previously described by Thomsen et al. [17].

\section{Statistical analysis}

Data was analyzed using descriptive statistics in STATA version 12 (StataCorp, College Station, TX, USA). The count of samples with mutant and wild type alleles were used to generate prevalence of the Pfmdr-1 N86Y and D1246Y alleles.

\section{Results}

Prevalence of the SNPs in the pfmdr-1 gene

Out of the 200 microscopically positive samples screened for Pfmdr-1 SNPs polymorphism (22 from ART and 178 from OPD clinics), $29.5 \%(\mathrm{n}=59 / 200)$ and $27.5 \%$ $(\mathrm{n}=55 / 200)$ contained the outer codons c86/c184 and $\mathrm{c} 1246$, respectively. $84.7 \%(\mathrm{n}=50 / 59)$ and $94.5 \%$ $(n=52 / 55)$ of these samples were genotyped at codons 86 and 1246 with the elimination samples which did not amplify in the nested PCR. Our results indicate a 90\% $(\mathrm{n}=45 / 50)$ prevalence of the wild type $86 \mathrm{~N}$ allele compared to $10 \%(n=5 / 50)$ of the mutant $86 \mathrm{Y}$ allele (Table 1$)$. Similarly, we observed a $92.3 \%(n=48 / 52)$ prevalence of wild 1246 alleles compared to $7.7 \%(n=4 / 52)$ of the mutant alleles in the study population (Table 1 ).

\section{Discussion}

We examined the prevalence of $P f m d r-1$ N86Y and D1246Y genotypes associated with altered drug sensitivity in febrile outpatients at LRRH utilising AL, the national treatment regimen. The prevalence of the wild type N86 and D1246 alleles were high in comparison to the mutant $86 \mathrm{Y}$ and $1246 \mathrm{Y}$ alleles (Table 1). The

\begin{tabular}{|c|c|c|c|c|}
\hline SNPs codon & Pfmdr-1 alleles & Examined & Positive & Prevalence \% \\
\hline \multirow[t]{2}{*}{ Codon 1246} & Wild (1246D) & 52 & 48 & 92.3 \\
\hline & Mutant (1246Y) & 52 & 4 & 7.7 \\
\hline \multirow[t]{2}{*}{ Codon 86} & Wild (N86) & 50 & 45 & 90.0 \\
\hline & Mutant (86Y) & 50 & 5 & 10.0 \\
\hline
\end{tabular}

SNPs: single nucleotide polymorphism, Pfmdr-1: P. falciparum multidrug resistance 1 gene 
widespread use of AL to treat malaria has increased both the prevalence and frequency of $P f m d r-1$ wild alleles N86 and D1246, with a decrease in the sensitivity to lumefantrine and increased sensitivity to aminoquinolines [18]. Our results correlate with the findings of increased prevalence of wild type N86 and D1246 polymorphisms associated with treatment failures and decreased sensitivity to lumefantrine in Tororo, Uganda $[19,20]$. Like in our study, a previous study by Okombo [21] in the neighbouring Kenya also observed a significant increase in the prevalence of wild type pfmdr1 N86/Y184/D1246 haplotype and a corresponding decline of the mutant pfmdr1 86Y/184Y/1246Y after 19 years (1995-2013) of chloroquine removal. This finding and ours highlight the importance of continued monitoring and characterization of parasite genotypes as a way to audit the therapeutic efficacy of drugs in clinical use and those previously withdrawn. The recurrent malaria infections prior to treatment with $\mathrm{AL}$ are associated with decreased lumefantrine sensitivity, and increased N86 genotypes could suggest significant risk factors for recrudescence in patients treated with $\mathrm{AL}[11,18]$. Interestingly, $\mathrm{AL}$ and AS/AQ exert opposing selective effects on the SNPs in Pfcrt and Pfmdr-1 genes [11].

By year 2000, the CQ resistance in one of the eastern districts in Uganda, were over 90\% [20]. In this study we found low prevalence of the mutant $86 \mathrm{Y}$ and $1246 \mathrm{Y}$ alleles which corroborates with previous findings of a gradual decrease in mutant $86 \mathrm{Y}$ and $1246 \mathrm{Y}$ genotypes in Uganda in between 2003 and 2012, following the withdrawal of CQ [20]. Prior to the withdrawal of CQ in Uganda, the CQ parasitological resistance levels ranged from $<5$ to $>50 \%$ [22]. The low mutant of $86 \mathrm{Y}$ and $1246 \mathrm{Y}$ genotypes could have resulted from the decreased use of CQ and widespread use of AL as the standard antimalarial drug in LRRH. A decreasing CQ exposure, and increased lumefantrine exposure selects for wild type sequences at these alleles $[16,23,24]$. Additionally, the low scale use of the $\mathrm{AS} / \mathrm{AQ}$, alternative first line drug for treatment of uncomplicated malaria could partly explain the low prevalence of the mutant alleles. Taken together, the current study suggests a prevalence rate of wild type alleles $1246 \mathrm{D}$ and $\mathrm{N} 86$ of about $90 \%$ compared with other studies in the region, which by 2012 was about $50 \%$ [20], suggesting high risk of treatment failures by AL. However, thus far, treatment of severe malaria in children below 20kg with intravenous AL was effective [25] indicating that, drug resistance to AL have not spread in the area.

In conclusion, our results demonstrated a high prevalence of wild type alleles N86 and D1246 that are likely to mediate decreased sensitivity to artemether-lumefantrine in the Lira RRH outpatients' clinics. However, the mutant
$86 \mathrm{Y}$ and $1246 \mathrm{Y}$ alleles showed a low prevalence owing to the decreased use of CQ and low use of AS/AQ, the alternative first line regimen in Uganda. A sustained success in malaria control is strongly dependent on the continued effectiveness of treatment using ACT, thus the need for continued countrywide monitoring of the Pfmdr-1 SNPs.

\section{Limitations}

We acknowledge limitations in the study including;

- The small sampling size involving only 771 out patients at LRRH participating in this study and small number of the resistance mediating polymorphism was considered.

- The possibility of sampling bias as a P. falciparum specific rapid kit was used for screening and existence of HRP2 and HRP3 mutant parasites.

- Low parasitemia could have led to potential false negative results especially in semi immune populations in the study area.

- The Afl-III enzyme used in genotyping could not recognize and digest both $86 \mathrm{Y}$ and $86 \mathrm{~F}$, and thereby unable to differentiate between them. However, the 86F genotype has only been reported in one study from Swaziland and not in Uganda or East Africa [26] thus all digestions with Afl-III were considered $86 \mathrm{Y}$ isolates.

\begin{abstract}
Abbreviations
ACT: artemisinin combination therapy; AL: artemether-lumefantrine; ART : anti-retroviral therapy; AQ: amodiaquine; DNA: deoxyribonucleic acid; IRB: Institutional review board; LRRH: Lira Regional Referral Hospital; PCR: polymerase chain reaction; Pfcrt: Plasmodium falciparum chloroquine resistant gene; Pfmdr-1: Plasmodium falciparum multi drug resistant gene-1; RFLP: restricted fragment length polymorphism; SNPs: single nucleotide polymorphisms.
\end{abstract}

\section{Authors' contributions}

$\mathrm{EA}, \mathrm{SO}, \mathrm{HE}, \mathrm{GMM}$ and RE conceived the study. EA collected data, performed experiments together with $\mathrm{HE}$ and later drafted the manuscript. All authors participated in analysis of the results. All authors read and approved the final manuscript.

\section{Author details \\ ${ }^{1}$ Department of Biology, Faculty of Science, Gulu University, P.O. Box 166, Gulu, Uganda. ${ }^{2}$ Department of Immunology and Microbiology, Gulu University, P.O. Box 166, Gulu, Uganda. ${ }^{3}$ Gulu University Bioscience Research Laboratories, P.O. Box 166, Gulu, Uganda. ${ }^{4}$ Department of Environmental and Biological Sci- ences, University of Eastern Finland, P.O. Box 111, 80101 Joensuu, Finland.}

Acknowledgements

We thank the study clients, management and staff of Lira RRH for the support rendered during the sample and data collection phase of this study.

\section{Competing interests}

The authors declare that they have no competing interests. 


\section{Availability of data and materials}

The authors declare that all the main data supporting the findings of this study are available within the article. Any additional data sets are available from the corresponding author upon reasonable request.

\section{Consent for publication}

Not applicable.

\section{Ethics approval and consent to participate}

This study was approved by the Institutional Review Board (IRB) of St Mary's Hospital Lacor, (No. LHIREC: 069/11/14). Permission to conduct this study was later granted by the management of Lira regional referral hospital and written informed consent and assent were obtained from the adult participants and caregivers, respectively.

\section{Funding}

This work was a students' funded project.

\section{Publisher's Note}

Springer Nature remains neutral with regard to jurisdictional claims in published maps and institutional affiliations.

\section{Received: 20 March 2019 Accepted: 15 April 2019}

Published online: 23 April 2019

\section{References}

1. WHO. World malaria report. 2018. ISBN 978924156469 4. 2018. http:// www.who.int/malaria. Accessed 22 Nov 2018.

2. USAID UPMI. President'S Malaria Initiative Angola. USAID. 2018;1-111.

3. Eyase FL, Akala HM, Ingasia L, Cheruiyot A, Omondi A, Okudo C, et al. The role of Pfmdr1 and Pfcrt in changing chloroquine, amodiaquine, mefloquine and lumefantrine susceptibility in Western-Kenya P. falciparum samples during 2008-2011. PLoS ONE. 2013;8(5):e64299.

4. Nanyunja M, Nabyonga Orem J, Kato F, Kaggwa M, Katureebe C, Saweka J. Malaria treatment policy change and implementation: the case of Uganda. Malar Res Treat. 2011;2011:1-14.

5. Rosenthal PJ. The interplay between drug resistance and fitness in malaria parasites. Mol Microbiol. 2013;89(6):1025-38.

6. Nosten F, White NJ. Artemisinin-based combination treatment of falciparum malaria. Am J Trop Med Hyg. 2018;77(6_Suppl):181-92.

7. Eastman RT, Fidock DA. Artemisinin-based combination therapies: a vital tool in efforts to eliminate malaria. Nat Rev Microbiol. 2009;7(12):864-74. https://ovidsp.ovid.com/ovidweb.cgi?T=JS\&PAGE=reference $\& D=$ emed9 $\& N E W S=N \& A N=2009600571$

8. Malmberg M, Ngasala B, Ferreira PE, Larsson E, Jovel I, Hjalmarsson A, et al. Temporal trends of molecular markers associated with artemetherlumefantrine tolerance/resistance in Bagamoyo district Tanzania. Malar J. 2013;12(1):1-7.

9. Ministry of Health (Uganda). The Uganda malaria reduction strategic plan 2014-2020. 2014:1-83.

10. Price RN, Cassar C, Brockman A, Duraisingh M, van Vugt M, White NJ, et al. The pfmdr1 gene is associated with a multidrug-resistant phenotype in Plasmodium falciparum from the Western Border of Thailand. Antimicrob Agents Chemother. 2018;43(12):2943-9.

11. Venkatesan M, Gadalla NB, Stepniewska K, Dahal P, Nsanzabana C, Moriera C, et al. Polymorphisms in Plasmodium falciparum chloroquine resistance transporter and multidrug resistance 1 genes: parasite risk factors that affect treatment outcomes for P. falciparum malaria after artemether-lumefantrine and artesunate-amodiaquine. Am J Trop Med Hyg. 2014;91(4):833-43.

12. Djimdé A, Doumbo OK, Steketee RW, Plowe CV. Application of a molecular marker for surveillance of chloroquine-resistant falciparum malaria. Lancet. 2001;358(9285):890-1.
13. Sisowath C, Petersen I, Veiga MI, Mårtensson A, Premji Z, Björkman A, et al. In vivo selection of Plasmodium falciparum parasites carrying the chloroquine-susceptible pfcrt K76 allele after treatment with artemetherlumefantrine in Africa. J Infect Dis. 2009;199(5):750-7.

14. Mwai L, Kiara SM, Abdirahman A, Pole L, Rippert A, Diriye A, et al. In vitro activities of piperaquine, lumefantrine, and dihydroartemisinin in Kenyan Plasmodium falciparum isolates and polymorphisms in pfcrt and pfmdr1. Antimicrob Agents Chemother. 2009;53(12):5069-73.

15. Otienoburu SD, Suay I, Garcia S, Thomas NV, Srisutham S, Björkman A, et al. An online mapping database of molecular markers of drug resistance in Plasmodiumfalciparum: the ACT Partner Drug Molecular Surveyor. Malar J. 2019;18(1):1-10. https://doi.org/10.1186/s12936-019-2645-x

16. Humphreys GS, Merinopoulos I, Ahmed J, Whitty CJM, Mutabingwa TK, Sutherland CJ, et al. Amodiaquine and artemether-lumefantrine select distinct alleles of the Plasmodium falciparum mdr1 gene in Tanzanian Children treated for uncomplicated malaria. Antimicrob Agents Chemother. 2006;51(3):991-7.

17. Thomsen TT, Ishengoma DS, Mmbando BP, Lusingu JP, Vestergaard LS Theander TG, et al. Prevalence of single nucleotide polymorphisms in the Plasmodium falciparum multidrug resistance gene (Pfmdr-1) in Korogwe District in Tanzania before and after introduction of artemisinin-based combination therapy. Am J Trop Med Hyg. 2011;85(6):979-83.

18. Tumwebaze P, Conrad MD, Walakira A, LeClair N, Byaruhanga O, Nakazibwe $C$, et al. Impact of antimalarial treatment and chemoprevention on the drug sensitivity of malaria parasites Isolated from Ugandan children. Antimicrob Agents Chemother. 2015;59(6):3018-30.

19. Conrad MD, Bigira V, Kapisi J, Muhindo M, Kamya MR, Havlir DV, et al. Polymorphisms in K13 and Falcipain-2 associated with artemisinin resistance are not prevalent in Plasmodium falciparum isolated from Ugandan children. PLOS ONE. 2014;9(8):e105690.

20. Mbogo GW, Nankoberanyi S, Tukwasibwe S, Baliraine FN, Nsobya SL, Conrad MD, et al. Temporal changes in prevalence of molecular markers mediating antimalarial drug resistance in a high malaria transmission setting in Uganda. Am J Trop Med Hyg. 2014;91(1):54-61.

21. Okombo J, Kamau AW, Marsh K, Sutherland CJ, Ochola-Oyier LI. Temporal trends in prevalence of Plasmodium falciparum drug resistance alleles over two decades of changing antimalarial policy in coastal Kenya. Int J Parasitol Drugs Drug Resist. 2014;4(3):152-63. https://dx.doi. org/10.1016/j.ijpddr.2014.07.003

22. Kamya MR, Bakyaita NN, Talisuna AO, Were WM, Staedke SG. Increasing antimalarial drug resistance in Uganda and revision of the national drug policy. Trop Med Int Health. 2002;7(12):1031-41. https://ovidsp.ovid.com/ ovidweb.cgi?T $=J S \& P A G E=$ reference $\& D=$ emed5\&NEWS $=N \& A N=20033$ 23419

23. Lobo E, De Sousa B, Rosa S, Figueiredo P, Lobo L, Pateira S, et al. Prevalence of pfmdr1 alleles associated with artemether-lumefantrine tolerance/resistance in Maputo before and after the implementation of artemisinin-based combination therapy. Malar J. 2014;13(1):1-6.

24. Dokomajilar C, Nsobya SL, Greenhouse B, Rosenthal PJ, Dorsey G. Selection of Plasmodium falciparum pfmdr1 alleles following therapy with artemether-lumefantrine in an area of Uganda where malaria is highly endemic. Antimicrob Agents Chemother. 2006;50(5):1893-5.

25. Byakika-Kibwika P, Nyakato P, Lamorde M, Kiragga AN. Assessment of parasite clearance following treatment of severe malaria with intravenous artesunate in Ugandan children enrolled in a randomized controlled clinical trial. Malar J. 2018;17(1):400. https://doi.org/10.1186/s1293 6-018-2552-6.

26. Dlamini SV, Beshir K, Sutherland CJ. Markers of anti-malarial drug resistance in Plasmodium falciparum isolates from Swaziland: identification of pfmdr1-86F in natural parasite isolates. Malar J. 2010;9(68):1-9. https:// www.malariajournal.com/content/9/1/68 